\title{
RADIOLOGY IN SYPHILIS*
}

\author{
BY \\ K. HARVEY GASKELL \\ Consultant Radiologist to Bristol Royal Hospitals \\ From the Department of Radiology, University of Bristol
}

Radiology finds useful application in almost all branches of medical science, in diagnosis, research, and teaching. In the daily medical approach to the diagnosis and cure of disease, the study of radiological features should frequently take us part of the way towards the understanding of an underlying pathological process. $X$ rays can often point to the site, extent, and type of a lesion, and indicate the body's reaction before or after the institution of treatment. Radiology should therefore frequently prove an aid to diagnosis and to control of treatment, but can seldom form the sole diagnostic investigation. Now that the expense of this equipment is squarely on our own shoulders, it is of economic importance to study true values, cutting out unavailing examinations and making wise use of staff and materials.

Our first lesson from closer radiological study is the wide range of normal anatomical variants to be found both in skeletal contours and in their densities. Our second lesson is a warning of the wide range of possibilities in regard to differential diagnosis which an abnormal shadow may signify. In correlating these shadows the radiologist must clearly tread in step with both clinician and pathologist if his reports are to be of value.

Study of syphilitic $x$-ray manifestations side by side with metabolic, neoplastic, and other inflammatory conditions is readily justified for these reasons :

(1) the clinical and $x$-ray pictures may be almost indistinguishable.

(2) the later lesions of syphilis are now uncommon and may be readily overlooked. This fact is surely a mute tribute to the efficient venereal clinic routine of the past two decades.

(3) unnecessary, sometimes mutilating, operations may be avoided as a result of deliberations upon an $x$-ray film.

* Based on an address given to the Medical Society for the Study of Venereal Disease, October 27, 1950.
In Bristol the radiological fraternity is fortunate in regularly seeing films of Dr. McLachlan's cases. His enthusiasm to have $x$-ray confirmation of diagnoses, and control films during treatment, has been a valuable aid in clinical and radiological teaching. His cases and those contributed by colleagues at the Bristol Royal Hospitals, including Prof. Neale, Dr. Sparks, and Dr. Middlemiss, compose the representative collection offered in this paper.

The scope of $x$-ray investigation in venereology includes bone syphilis, cardio-vascular syphilis, and alimentary syphilis, to which should be added the exclusion of intercurrent diseases. I do not propose to extend the paper by including the $x$-ray demonstration of gonococcal strictures, or lymphogranuloma inguinale.

\section{BONE SYPHILIS}

First let us consider this condition in general. The blood-borne infection seeds in the most vascular parts. Osteochondritis and periostitis are therefore the characteristic lesions in the infant and juvenile, and an osteoperiostitis in the adult. Bone can only react, as judged broadly from a radiograph, in two ways :

(a) Local densening from reactionary hyperostosis, or scar formation laid down as a result of any natural or therapeutic response in infected zones of bone.

(b) Local translucency in bone, first from demineralization shown as decalcification in the hyperaemic stage, merging later into destructive bone erosion if virulence of infection swamps local reaction.

On the radiograph these two contrasts in bone density may be seen in close apposition where a zone of hyperaemia (translucency) surrounds a granulomatous (dense) area.

Congenital Syphilis.-The radiograph will depend both on the duration of infection (infant's age when $x$-rayed) and any previously instituted maternal or infantile treatment. 
Metaphysitis, Diaphysitis.-The characteristic zone of terminal bone densening, metaphysitis, borders upon a translucent band of hyperaemia, thrown into sharp contrast in the contiguous portion of the affected long bones. These changes, originating during intra-uterine life, progress, if untreated, during the first dozen or more weeks of infancy into a destructive erosion of the bone shaft (diaphysitis). Wimberger's sign depends upon this feature of the disease; such erosion is frequently seen in the tibial condyle, neck of humerus, or femur.

Epiphysitis, Periostitis.-Clinically epiphysitis occurs pari passu with metaphysitis and periostitis, the former being visible on the radiographs only as a soft tissue swelling around and beyond the bone end where the epiphysis is not yet ossified.

Healing.-Anti-specific treatment first appears to abolish the visible translucent band of hyperaemia. The metaphysis then increases in density, broadens, and later, after therapy is completed, diminishes : but the final density cast by this once diseased area is proportional to the amount of residual scar, excess heavy metal, or both. Ultimate deformity of bone shape depends upon the degree of irreversible damage incurred by the growing epiphysis and periosteum during the invasive period of the disease, and upon weightbearing on softened bones in the juvenile group. These features may be followed from a study of serial radiographs illustrating congenital syphilis.

\section{$X$-Ray Features of Congenital Syphilis}

Case 1. Male infant, aged 6 weeks.-At the age of 3 weeks he cried when picked up, at 6 weeks he was pale, fretful, and off feeds.

Examination.-Knees, ankles, and wrists enlarged and tender. General adenitis. No visceral lesion. Skin dry, peeling over abdomen. Rash.

Wassermann reaction ++ ; Kahn test ++ .

Father sero-negative. Mother sero-positive.

Radiographs (Fig. $1 a$ and $b$ ).- All the long bone ends show terminal increased density of metaphysis and an underlying hyperaemic band of translucency. Periostitis is present along ulnae, radii, humeri, and leg bones. Under the lower femoral epiphyses the ossifying zones are irregular, and destructive erosion extends up into the medial condyles (diaphysitis). Note also the right testicular enlargement.

Case 2. D.S., infant, aged 5 weeks. - This case illustrates reactions to treatment at the right wrist joint. At 2 weeks he was fretful and refused feeds. At 3 weeks the wrists, etc., were swollen.

Examination.-At 5 weeks we found Wassermann reaction ++ , Kahn test +++ . Condylomata, etc., were present. Anti-specific treatment was given : penicillin 30,000 units 3-hourly for 18 days, then a 5 -week course of sulpharsphenamine.

Fig. 2(a) (5 weeks).-Shows swelling of epiphysis (soft tissue : centre not yet ossified). Terminal density (metaphysitis). Adjacent band of bone hyperaemia.

Fig. 2(b) (1 month later).-Shows reformed bone at hyperaemic layer after therapy, also ? reactionary

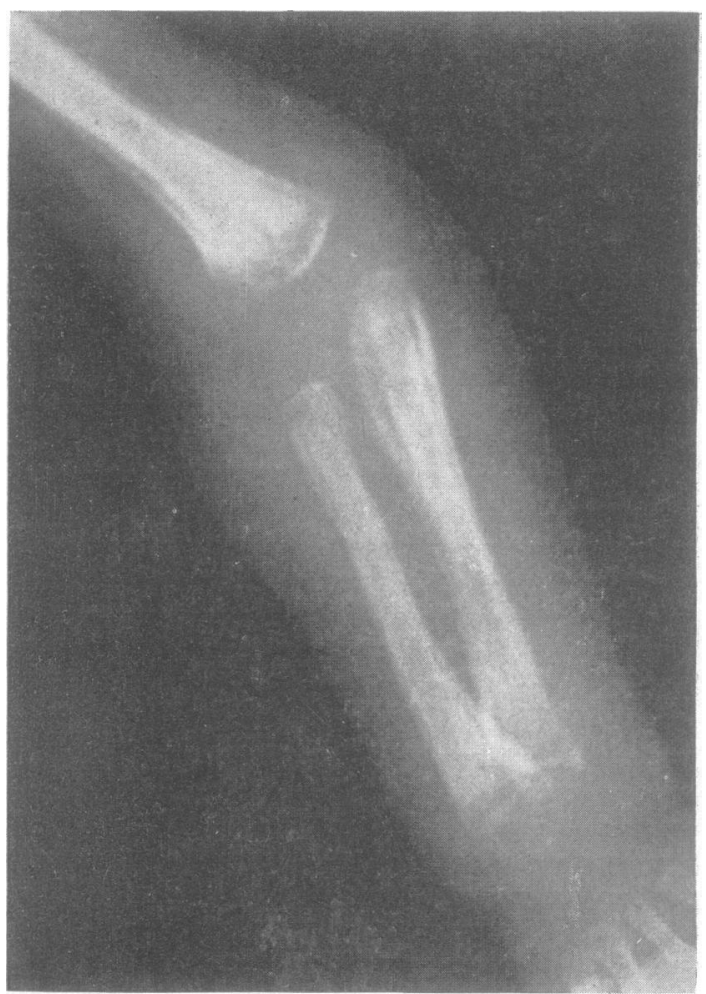

Fig. 1 (a).-Case 1, Periostitis, etc., in humerus, radius, and ulna.

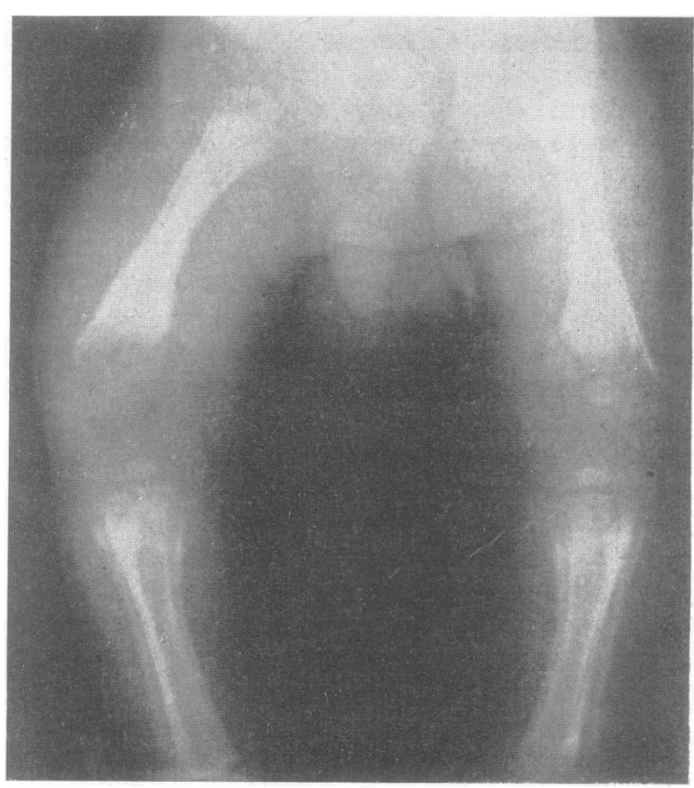

Fig. 1 (b).-Case 1, Periostitis in leg bones. 


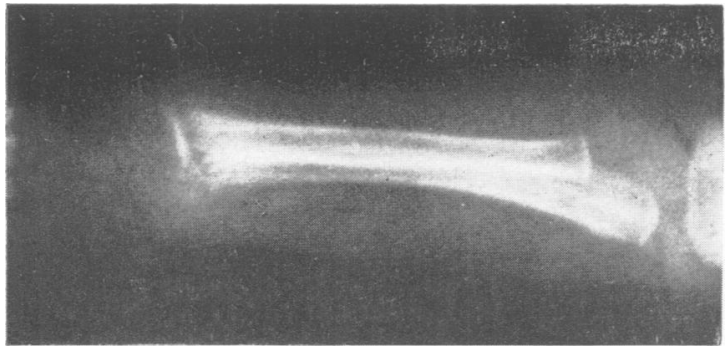

FIG. $2(a)$.-Case 2 at 5 weeks (before treatment).

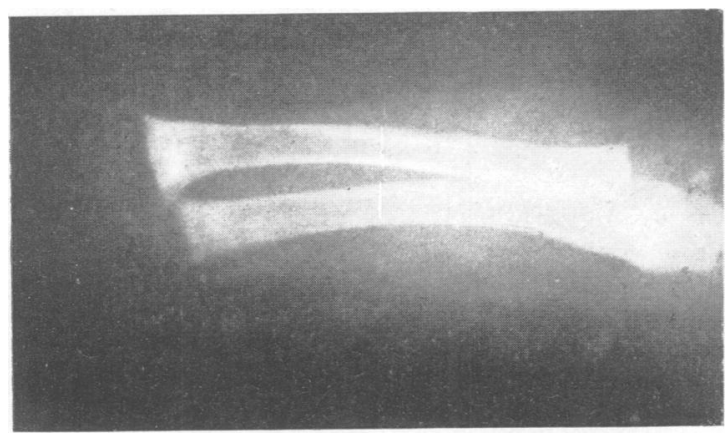

Fig. $2(c)$.-Case 2 at 27 weeks.

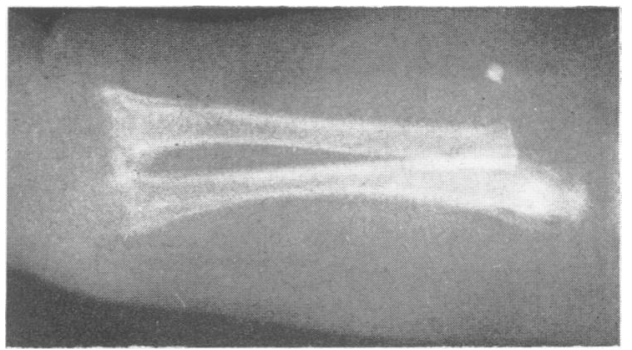

Fig. $2(b)$.-Case 2 at 9 weeks.

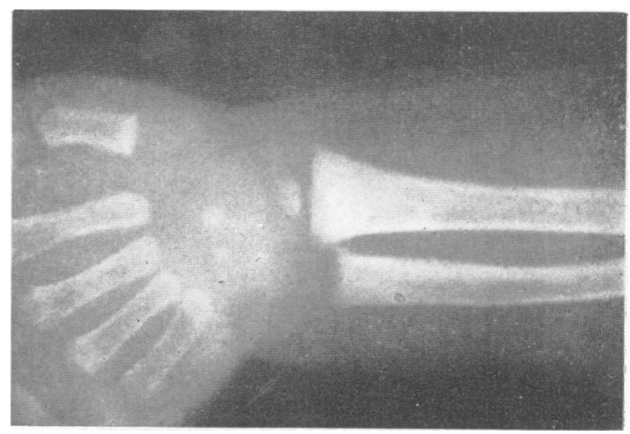

FIG. $2($ d). - Case 2 at 9 months (after disease arrest).

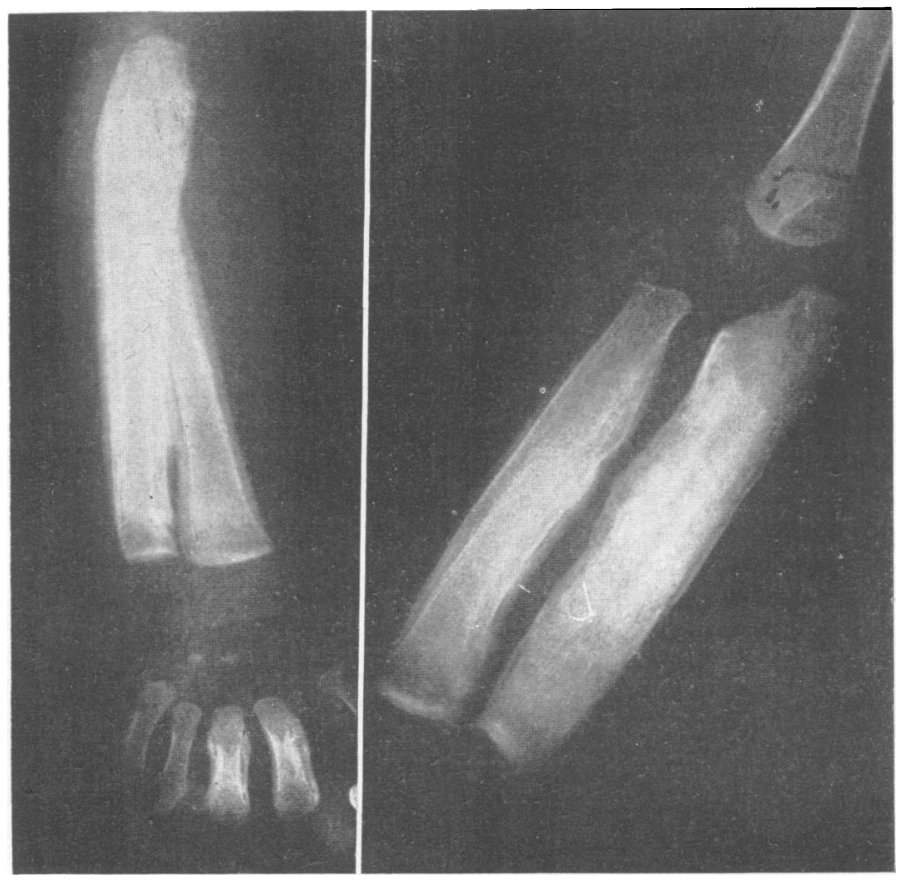

Fig. 3.-Case 3, extensive osteo-periostitis. periostitis. There is now broadening and " cupping" of terminal ossifying zone strongly suggesting early developing rickets.

Fig. 2(c) (4 months later).-Shows almost complete regression of latter changes, also of the ? reactionary periostitis. Note the residual scar density of previous metaphysitis now buried behind the growing end of bone.

Fig. 2(d) (3 months later).-After the appearance of lower radial epiphysis. Further bone sclerosis, after arrest of disease, is probably heavy metal deposit.

Case 3. J. P., infant aged 10 weeks.This case illustrates untreated metaphysitis with an extreme degree of osteoperiostitis (diffuse cortical hyperplasia). Note also the lesions of dactylitis, which Holt claims to be more common in the metacarpals and metatarsals, in the first year of life, than in the phalanges (Fig. 3).

No good example can be included of the final and rarer form of generalized rarefying osteitis which, owing to the ready occurrence of fractures in infancy, may simulate osteogenesis imperfecta. 


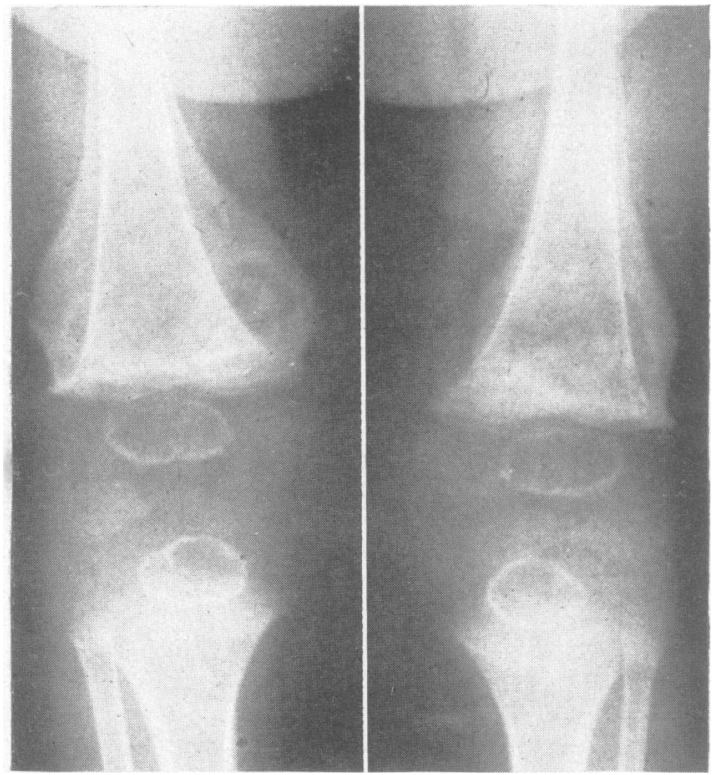

FIG. 4.-Case 4, infantile scurvy.

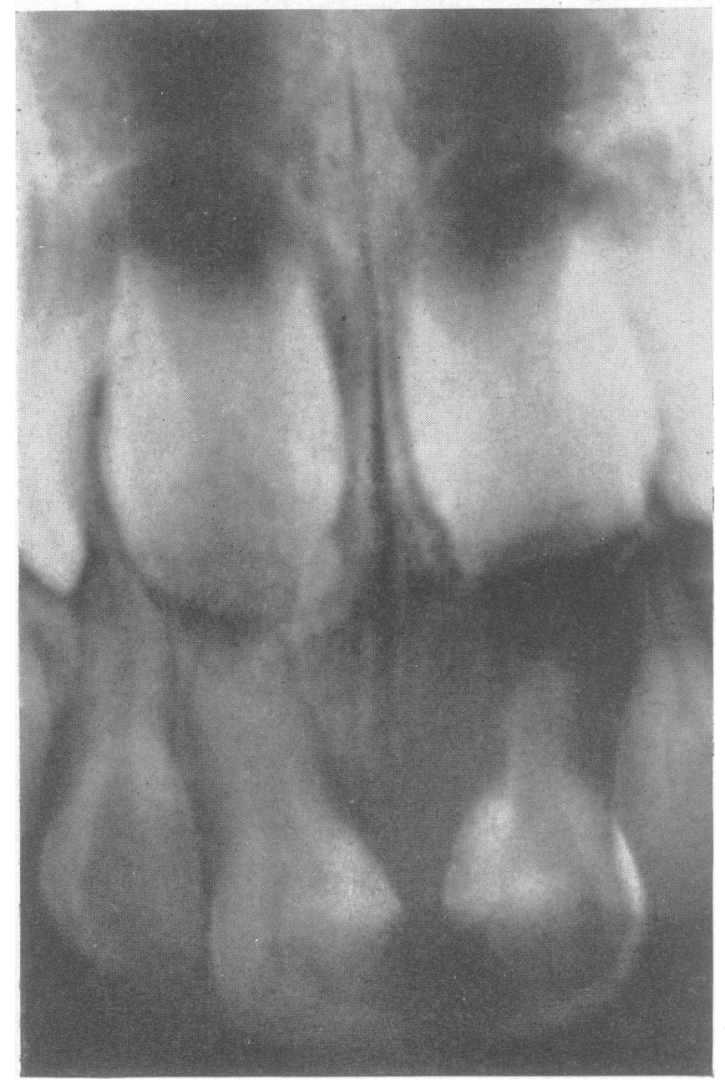

FIG. 5.-Case 5, Hutchinson's teeth.

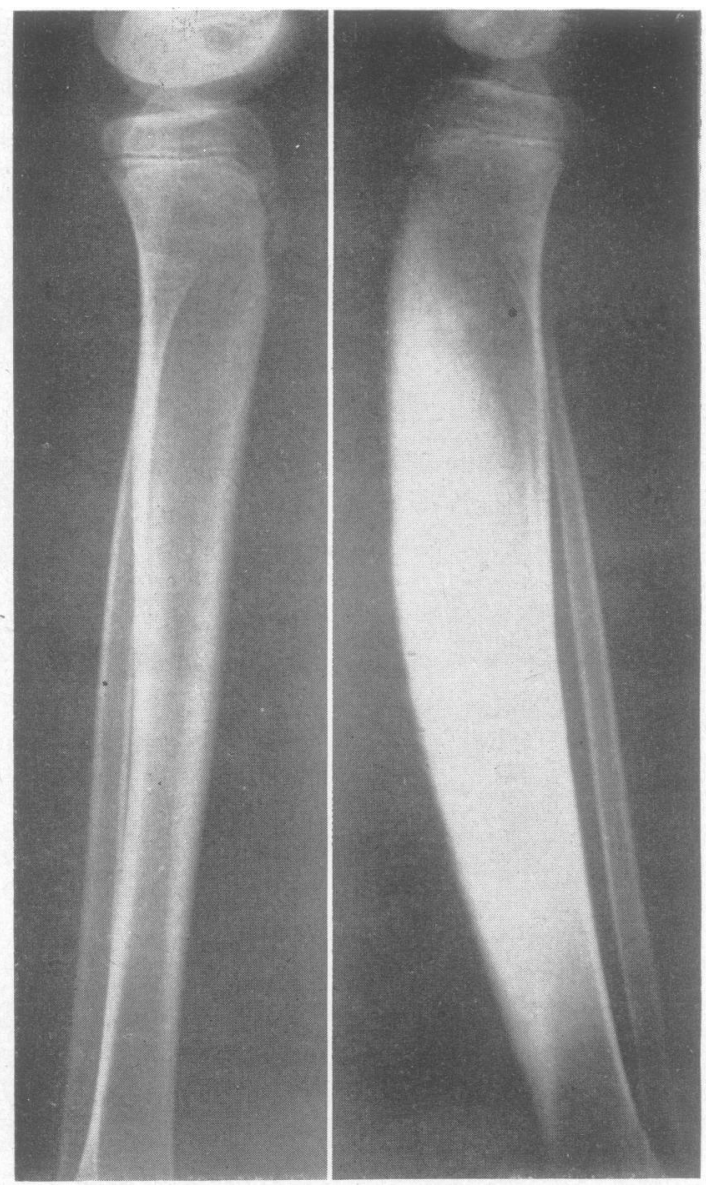

Fig. 6.-Case 6, lateral view of both legs, right leg sabre deformity.

Case 4. A. A., infant aged 18 months (Fig. 4).-This case of infantile scurvy is included for comparison of :

(a) age of occurrence.

(b) characteristic epiphyses, translucent as groundglass, showing egg-shell density of the cortex.

(c) massive sub-periosteal "soft" haemorrhagic shadow, in contrast with the "layer" or "lacework" formations which characterize periostitis.

(d) hyperaemic "bands" in femoral shafts and apparent densening of metaphyses (deep to the epiphyses) which may simulate the appearances of syphilitic infection.

Case 5.-This is an example of Hutchinson's teeth. Fig. 5 shows an occlusal dental $x$ ray of the central portion of the upper jaw. The erupted central (deciduous) teeth show erosion from caries : the permanent upper central incisors show the characteristic denticle defects revealed before tooth eruption. Hutchinson's teeth occur in a proportion only of congenital syphilitics, and under the age of 7 years $x$ rays may assist in the confirmation of diagnosis. 
$X$-Ray Features of Late Congenital Syphilis. The same underlying bone changes are found in juveniles as in the infantile manifestations. Modified lesions of the gummatous osteomyelitis type are also found; as in adults trauma appears to be a potent precipitating agent. Weight bearing during the hyperaemic stage of bone softening tends to increase juvenile deformities of the involved long bones, a factor which is non-operative in the infantile case. Three examples of juvenile disease follow :

Case 6. J. W., aged 11 (imbecile).-This case presented as "acute osteomyelitis" with acute pain having nocturnal exacerbations, etc. Urgent operative intervention was undertaken, the diagnosis being made from biopsy material and later confirmed serologically.

The right leg (Fig. 6) shows diffuse cortical thickening of the diaphysis with sabre shin deformity, and bowing consequent upon previous bone softening. There was a history of "some previous anti-syphilitic treatment" in infancy but no records were available.

Case 7. "S," aged 6 years.-Clinically the original suspicion was of sarcoma of the right leg. Fig. 7(a) shows diffuse osteo-periostitis of the right leg with areas of bone destruction in the fibula. The tibia shows

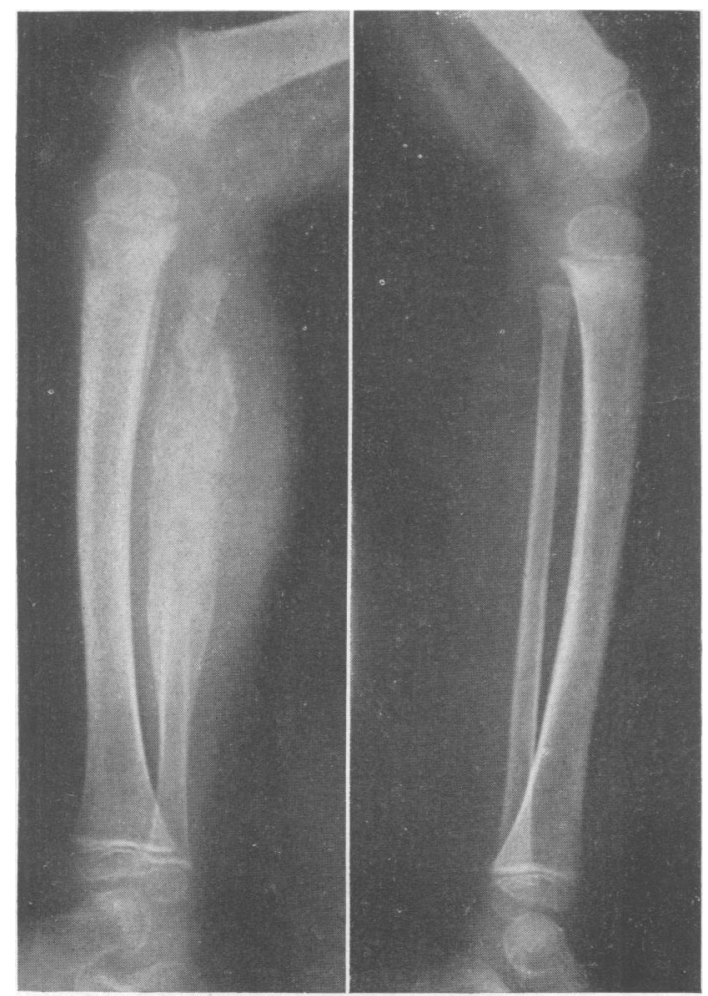

FIG. $7(a)$.-Case 7 , right and left legs, before treatment.

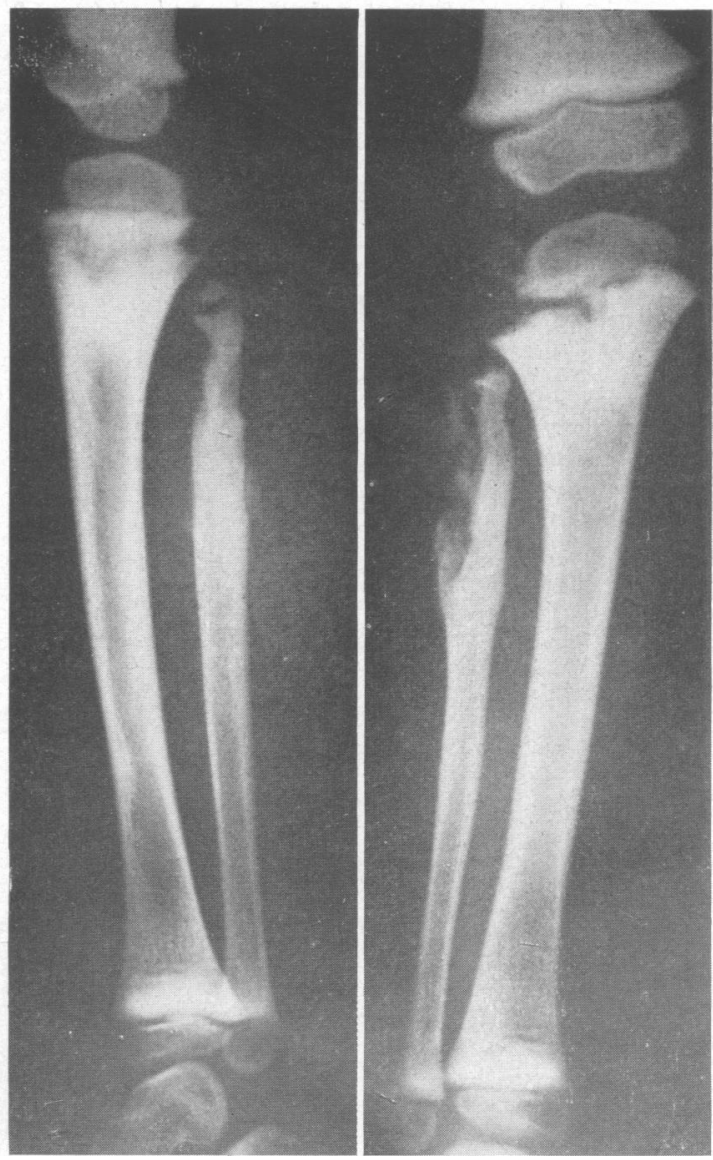

FIG. 7 (c).-Case 7, anterior and lateral views at stage of disease arrest. Note the Harris' lines of previous nutritional disturbance in the lower diaphysis, and the greatly increased metaphysical density at the termination of intensive heavy-metal treatment.

periostitis (localized) and destructive erosion of the upper tibial diaphysis causing collapse deformity.

. Metaphysitis of the upper end of the tibia of the left leg is seen with some associated bone collapse. There is diffuse osteo-periostitis of the lower end of the femoral diaphysis.

Fig. 7(b) illustrates the left knee and lower end of the left femur during treatment (overleaf).

Fig. 7(c) shows reduction of osteo-periostitis, and densening of the femoral metaphysis and erosive destruction at the outer angle of the tibial metaphysis.

The radiographs show the full therapeutic effect obtainable to date. Having regard to the original extent of bone loss under both tibial metaphyses, some final deformity may persist and be accentuated by weight bearing. 


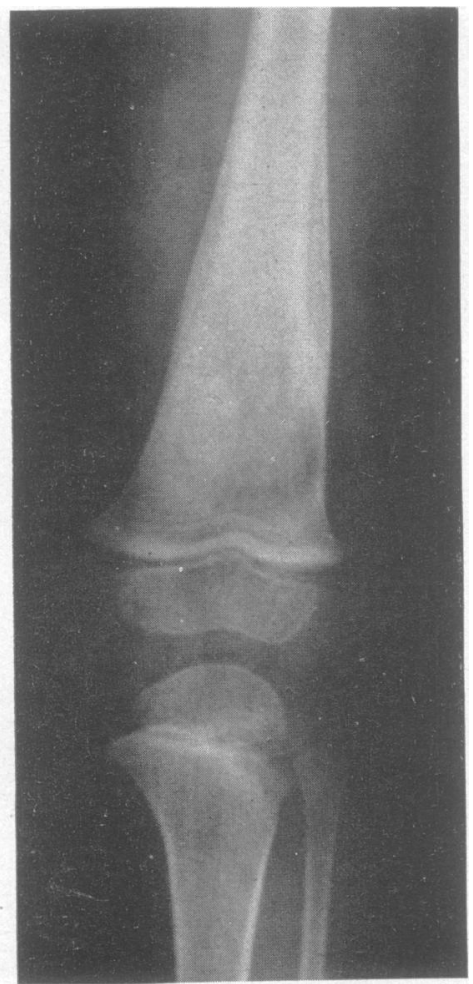

Fig. $7(b)$.-Case 7, left knee and femur, early in treatment.

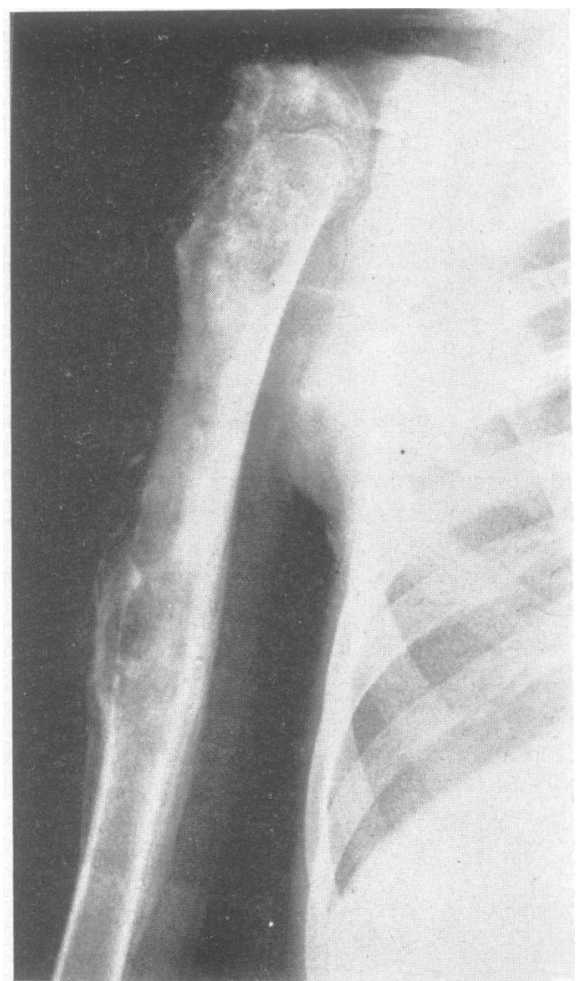

FIG. 8.-Case 8, gummatous osteomyelitis of upper half of humerus.
Case 8.-This example of osteomyelitis of the upper half of the humerus following local trauma shows no special radiological features to identify the underlying pathology (Fig. 8).

The lesion to be seen in the head of the humerus was originally regarded as tuberculous in spite of osteoperiostitis and osteoscopic pain at night. Congenital syphilis was not suspected until the onset of interstitial keratitis. The patient's serum and family history both proved to be positive.

Case 9. Aged 10.-This patient was referred for the venereologist's opinion, suspected of suffering from syphilitic dactylitis. At this time a single Kahn test returned an equivocal result.

Fig. 9 shows a chronic sclerosing osteomyelitis with bone cloacae in the affected phalanx, unlikely to be specific.

The therapeutic test, carried out with penicillin, arsenic, and bismuth, proved negative, and repeated serum tests were also negative. A diagnosis was therefore made of chronic non-specific osteomyelitis.

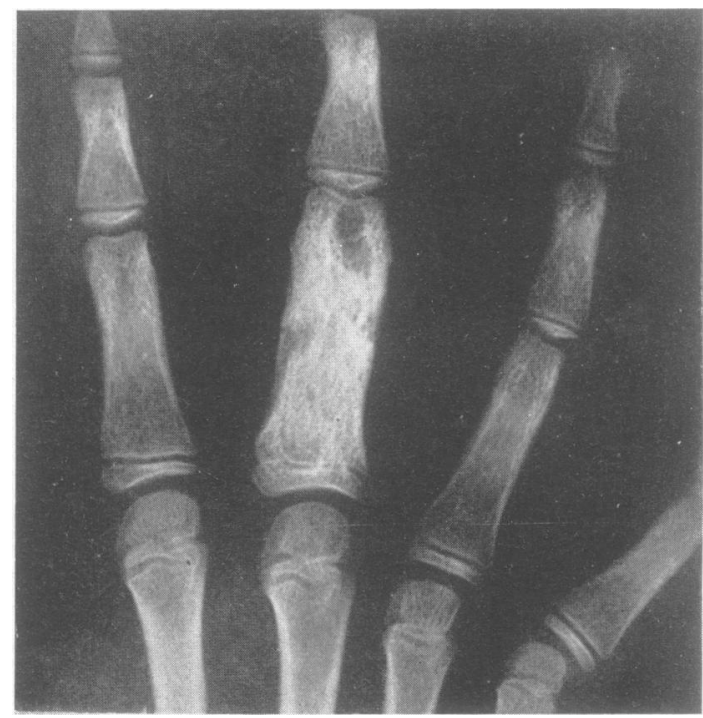

FIG. 9.-Case 9, first phalanx, middle finger. 


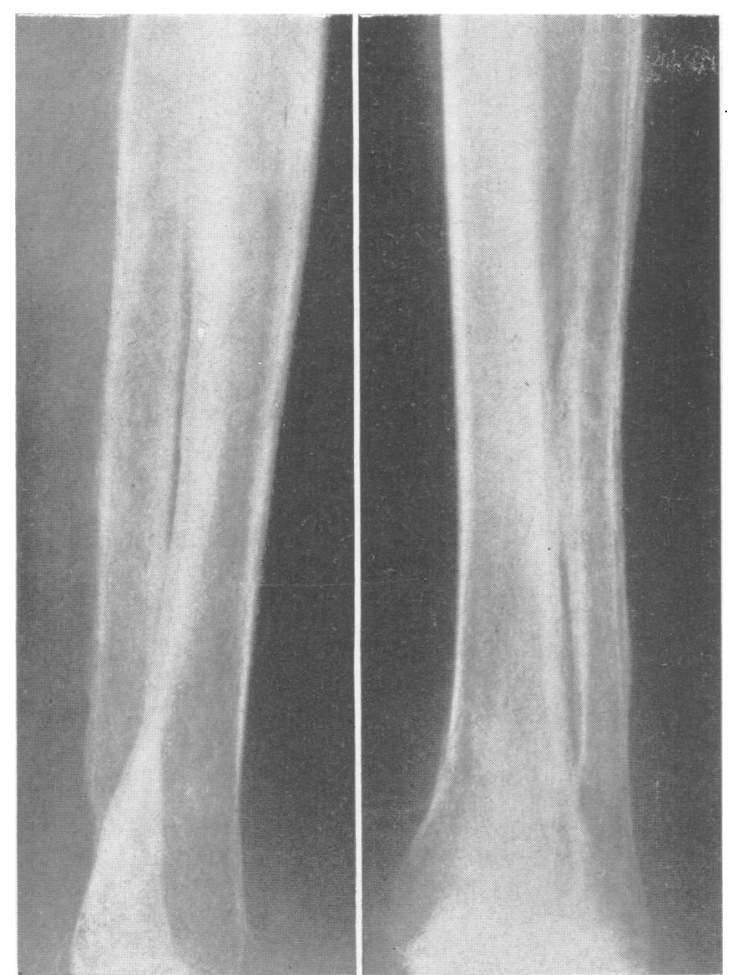

FIG. 10.-Case 10, periostitis in syphilis.

$X$-Ray Features of Adult Bone Syphilis.-In a demonstration of this nature it is the proven cases of non-syphilitic lesions which provide interesting and instructive material.

\section{Periostitis}

Case 10 (Fig. 10) is an example of syphilis.

Case 11 (Fig. 11) is an example of yaws.

Both conditions exhibit characteristically the lacework formation with coarse spiculation, to be contrasted, when localized, with the fine spicules sometimes seen in osteogenic sarcoma. The linear type of periostitis may be of syphilitic, non-specific inflammatory, or reactionary origin.

Case 12. A. F., male, aged 52.-This case is of interest from the differential point of view. The patient, a miner, came to hospital for orthopaedic assessment following union of a compound fracture of the left leg (Fig. 12a).

Contrary to the Medical Board's judgment he claimed to be incapable of work; he had tried to work but complained of pain in the left knee and leg. $X$ ray of the damaged leg showed a good union in a good position, but periosteal changes, well removed from the site of old fracture, called for a wider survey of the case.

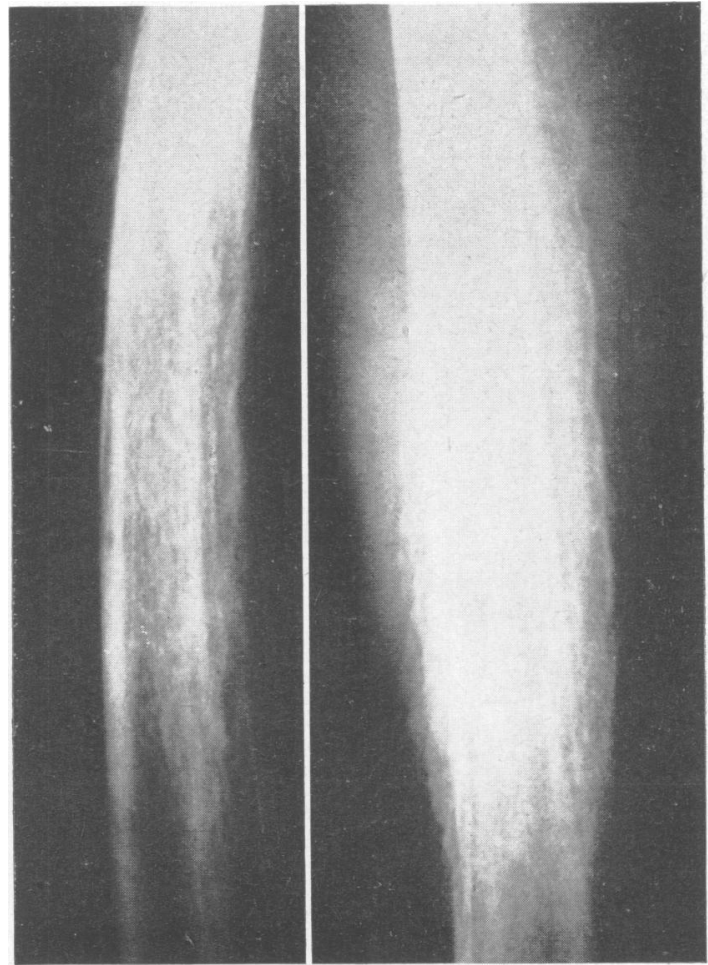

FIG. 11.-Case 11, periostitis in yaws.

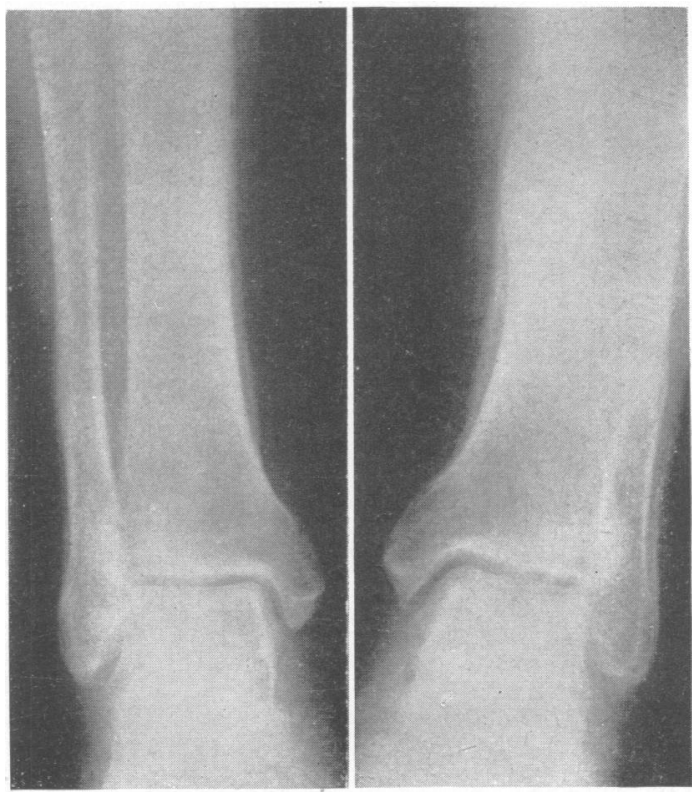

FIG. $12(a)$.-Case 12, periosteal changes in legs; old fracture in left leg. 


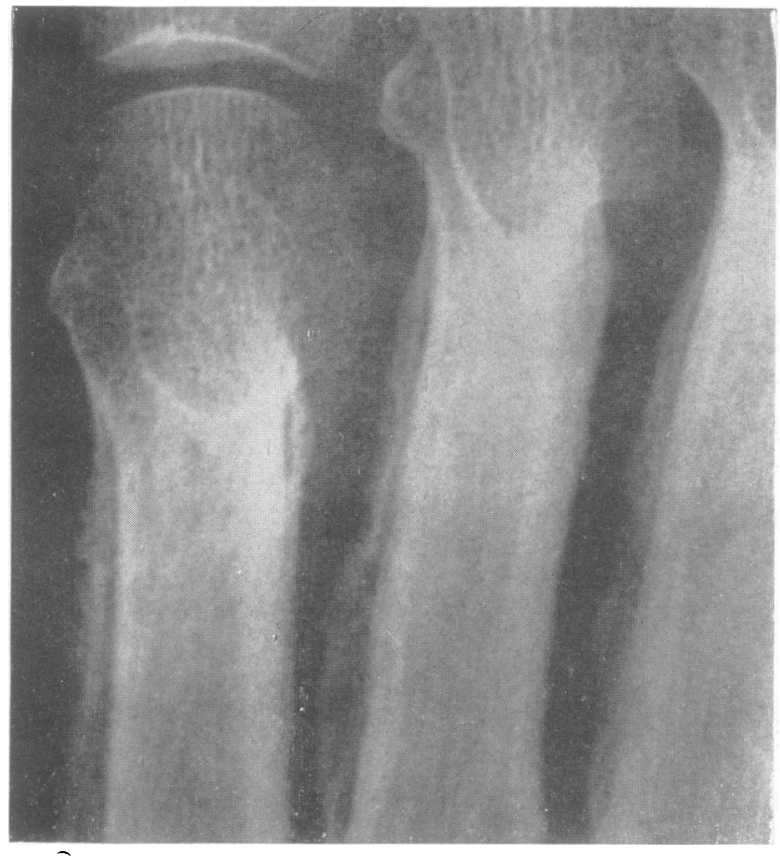

Fig. 14 (opposite) shows the left leg in anterior and lateral views.

If an opinion had been given regarding the thin anterior view, syphilis might have been suspected. Dense bones require increase of radiographic factors for their proper exploration.

Case 15.-This is an example of a gummatous ulcer of the leg with underlying periostitis. Suitable radiographic factors have been selected for this case, and demonstrate tangentially both the punched-out ulcer and the area of syphilitic osteitis of the tibia beneath (Fig. 15).

In this case healing was obtained in 4 weeks under anti-syphilitic treatment.

Local periostitis, deep to chronic varicose ulcers, is frequently encountered and is not thought to have any significance in this connexion.

Neuropathic Joints.-Examples of Charcot joints, although devoid of syphilitic bone features, must be included in any survey of syphilis, though it is of no credit either to

FIG. $12(b)$.-Case 12, periosteal changes in other bones.

These periosteal changes were then found in both femora, tibiae, fibulae, metacarpals, and metatarsals (Fig. 12b).

The presence of hypertrophic pulmonary osteopathy was confirmed when a silent lesion was revealed in the radiograph of the chest (Fig. 12c).

Blood serology was negative. Clinically, finger clubbing was minimal.

Osteitis.-The most interesting comparisons with lues are found in cases of deforming osteitis (Paget's disease).

Case 13. E. C., aged 61.-This patient had raised serum alkaline phosphatase and negative blood.

Fig. 13 shows the sabre-like tibia in lateral view.

Case 14. M. H., aged 58.-The diagnosis of Paget's disease in this patient was again supported by serum phosphatase and blood tests.

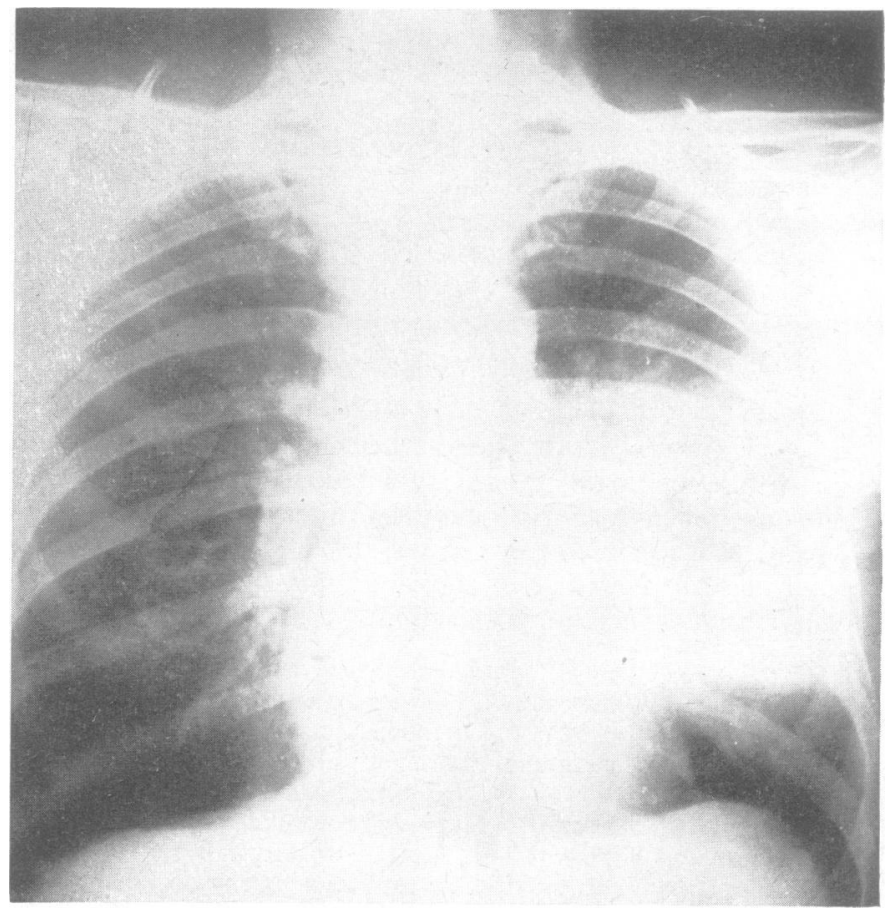

Fig. $12(c)$.-Case 12, chest radiograph. 


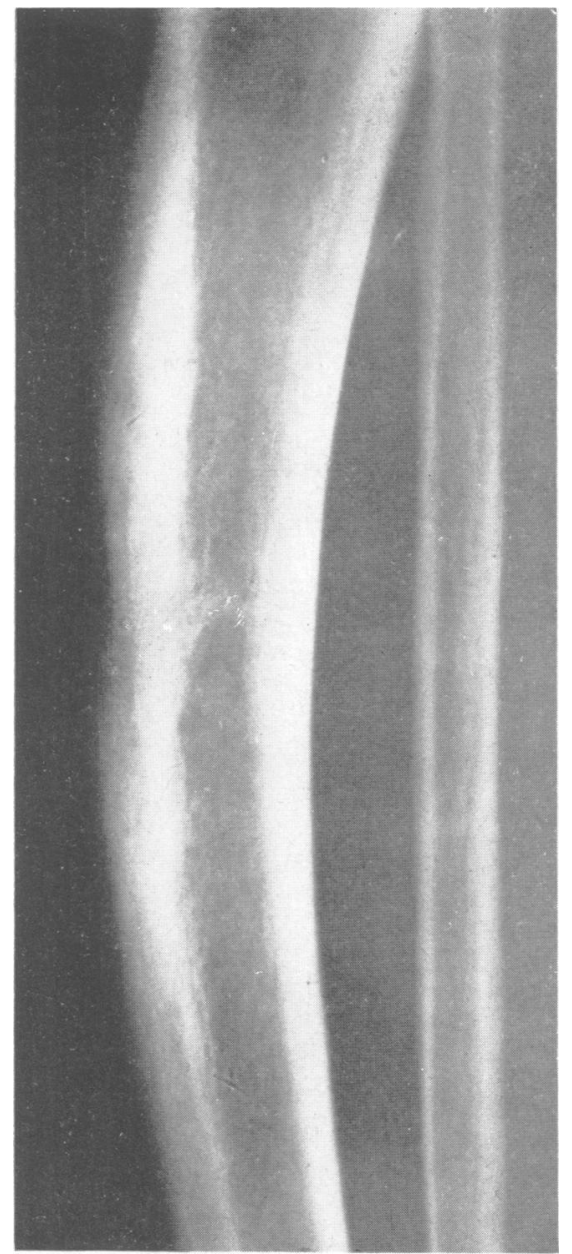

Fig. 13.-Case 13, osteitis deformans.

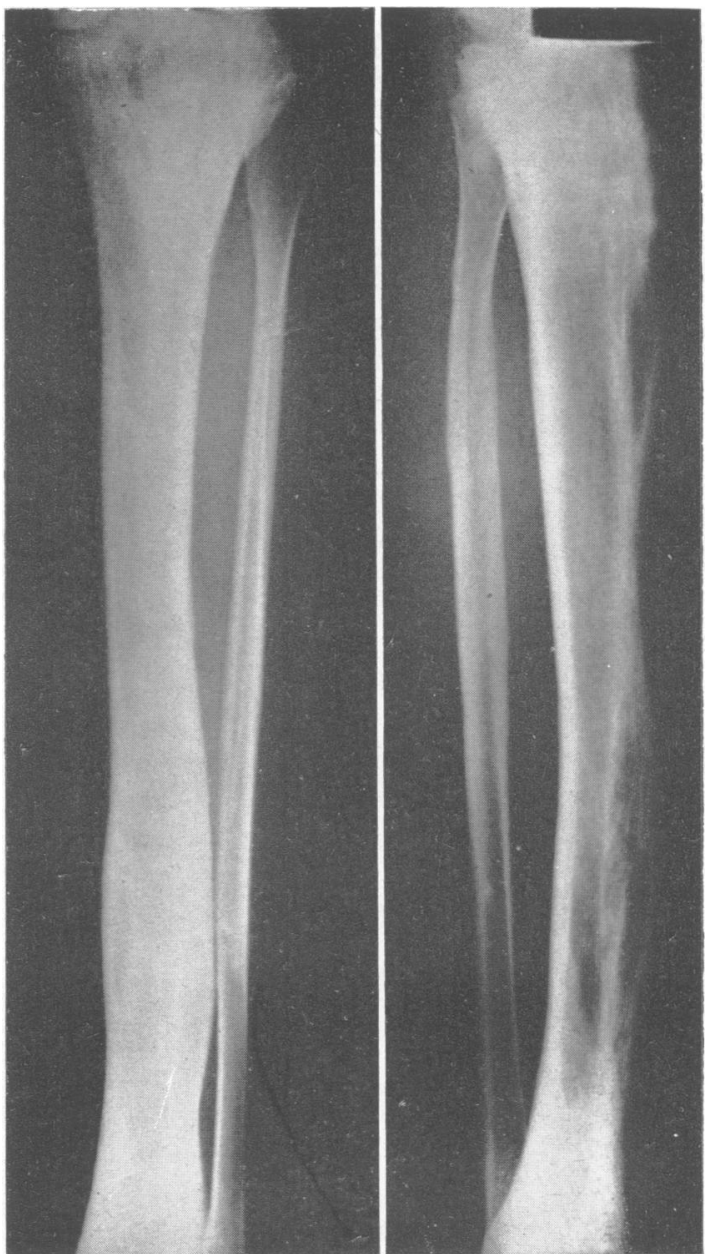

Fig. 14.-Case 14, anterior and lateral views of left leg.

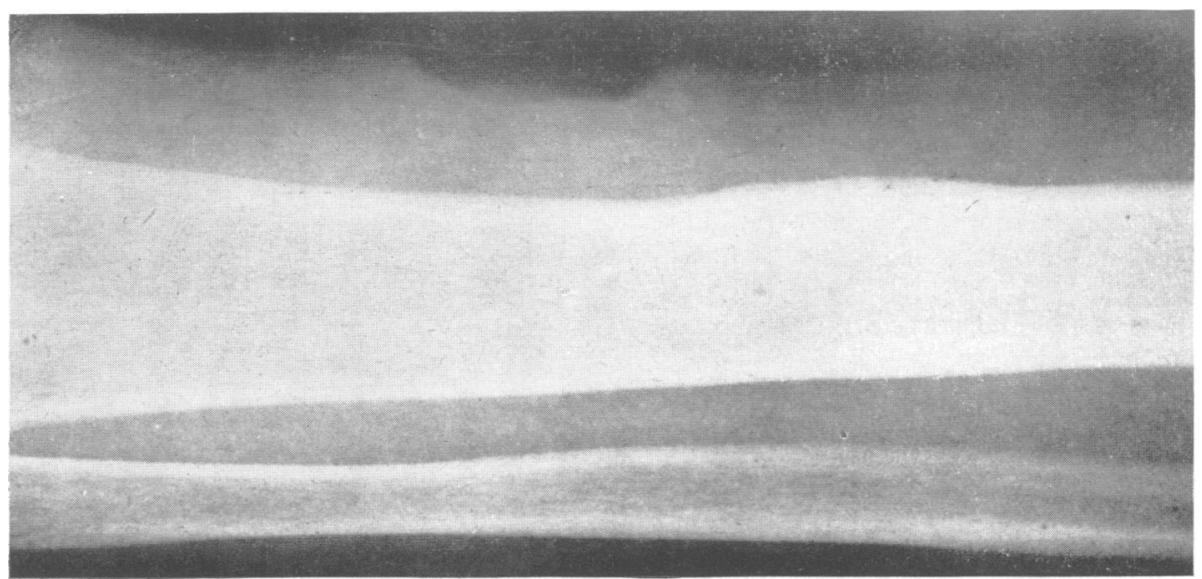

Fig. 15.-Case 15, gummatous leg ulcer with underlying periostitis. 
clinical medicine or to radiology to show advanced neuropathic joint cases.

When considering arthropathies, radiology should frequently be able to contribute materially to an understanding of the underlying aetiology and pathology, thereby assisting treatment. I can recollect, in my earlier days, reporting bluntly " destructive arthritis of knee" of a case in which further films could have revealed similar sarcoid deposits elsewhere in characteristic sites. Fortunately for this patient an orthopaedic surgeon explored his knee, made a diagnosis, and excised the deposits.

The following three cases of arthritis were found in known tabetic cases :

Case 16.-Hypertrophic Charcot of knee (Fig. 16).

Cases 17 and 18.-Atrophic Charcot of toe joints (Fig. 17) and hip joint (Fig. 18). In spite of destruction and mechanical disorganization the hip case walked moderately well.

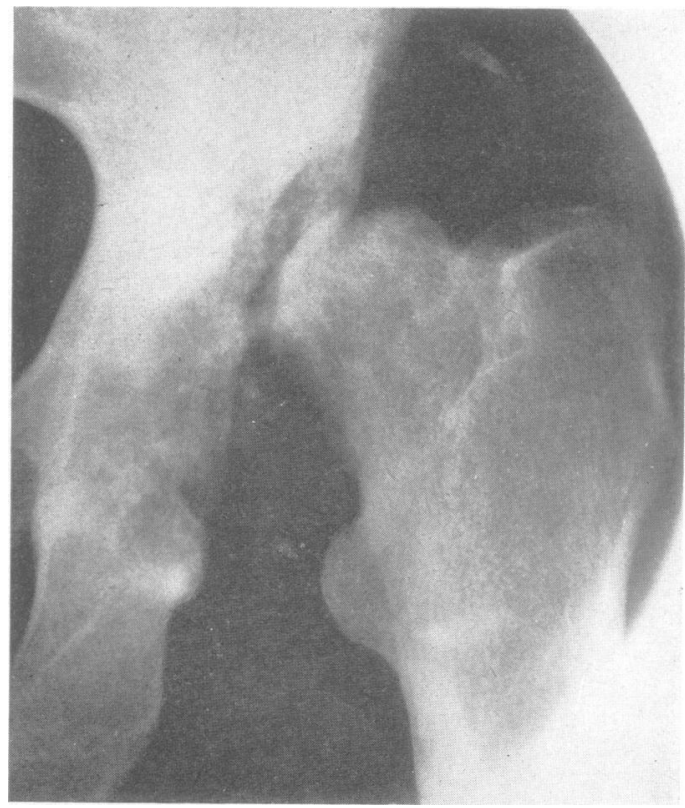

FIG. 18.-Case 18, atrophic Charcot of hip-joint.

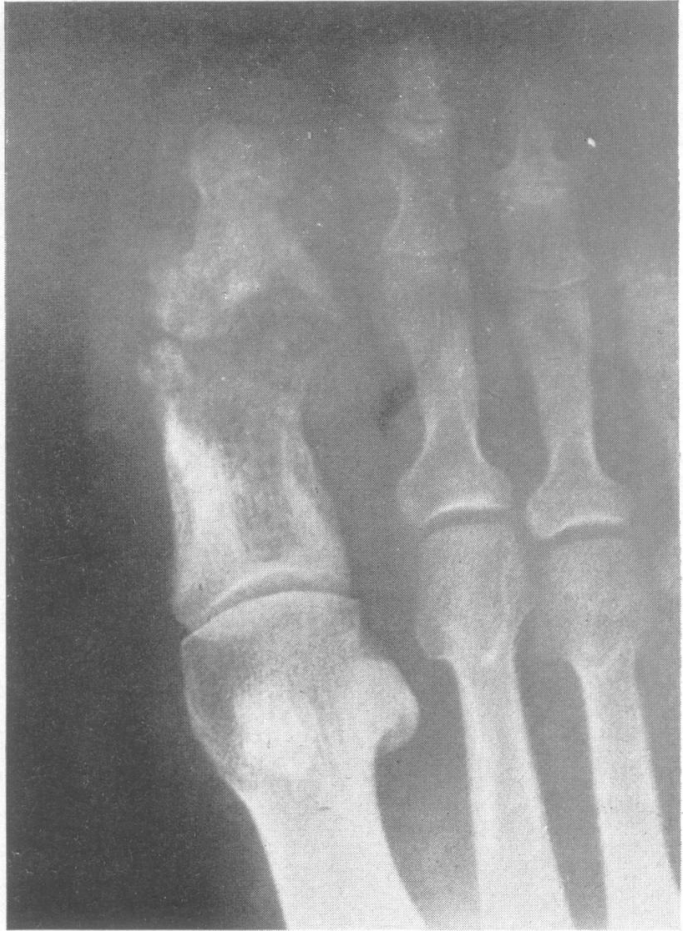

Fig. 17.-Case 17, atrophic Charcot of great-toe joint.

Fig. 16.-Case 16, hypertrophic Charcot of knee. 


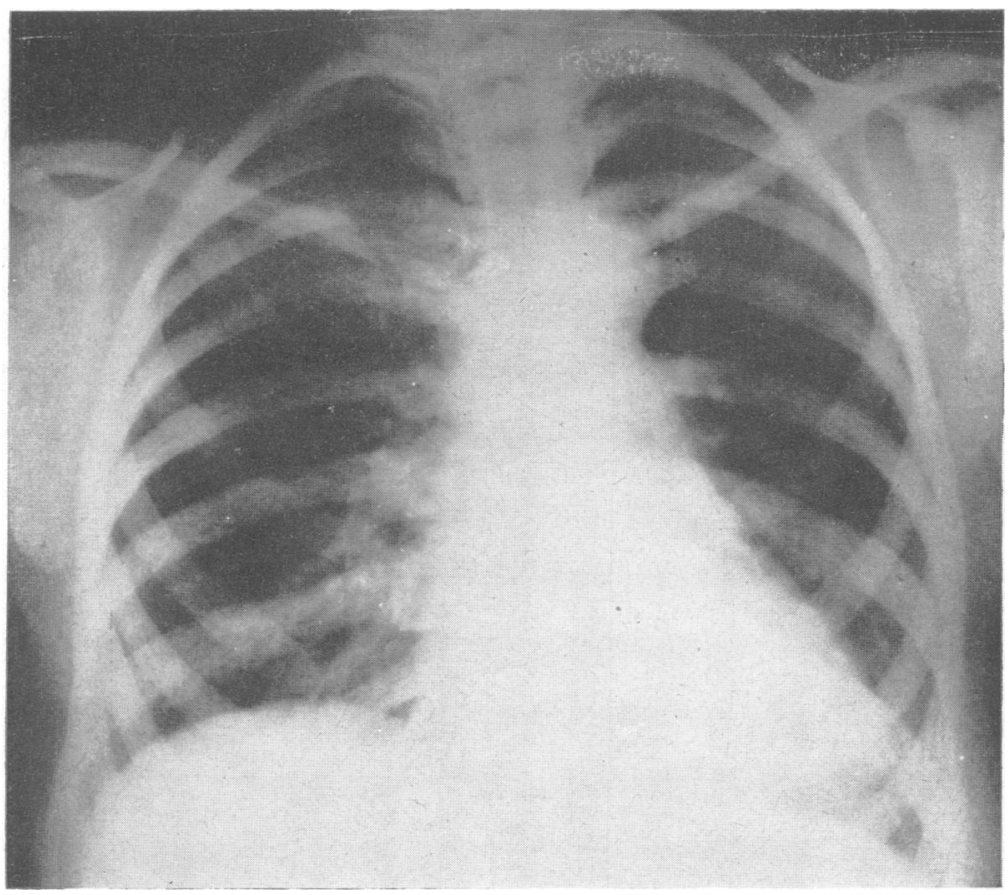

Fig. 19.-Case 19, rib, following prolonged heavy-metal therapy.

Other Abnormal Bone Densities.An interesting similarity between abnormal rib densities is shown in the two cases which are illustrated on this page :

Case 19. (Mrs. V.)-This patient was a known luetic in 1930, between which time and 1944 she received total doses of arsenic 79 g., bismuth 85 g., and colloidal mercury sulphide $50 \mathrm{ml}$.

Her bones have not since then given up their store, nor has her Wassermann reaction reverted to positive (Fig. 19).

Case 20 (Mr. G.).-This patient had unusually widespread deposits of sclerosing prostatic cancer metastases in all his bones.

The extension of the disease was relentless in spite of stilboestrol therapy (Fig. 20).

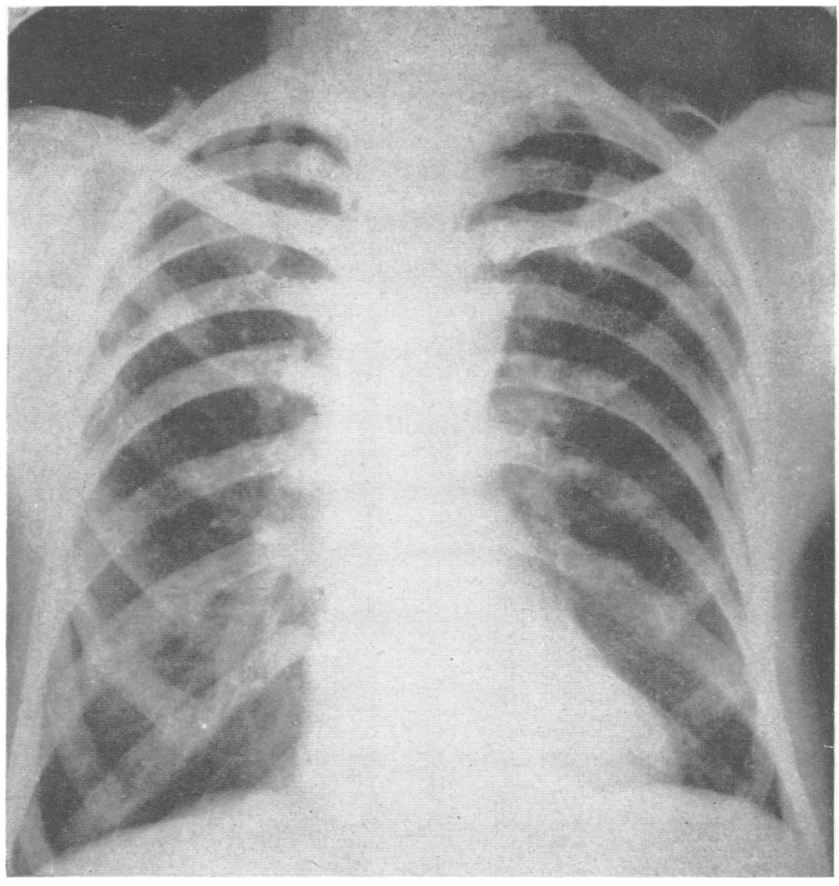

FIG. 20.-Case 20, ribs containing sclerosing cancer deposits. 


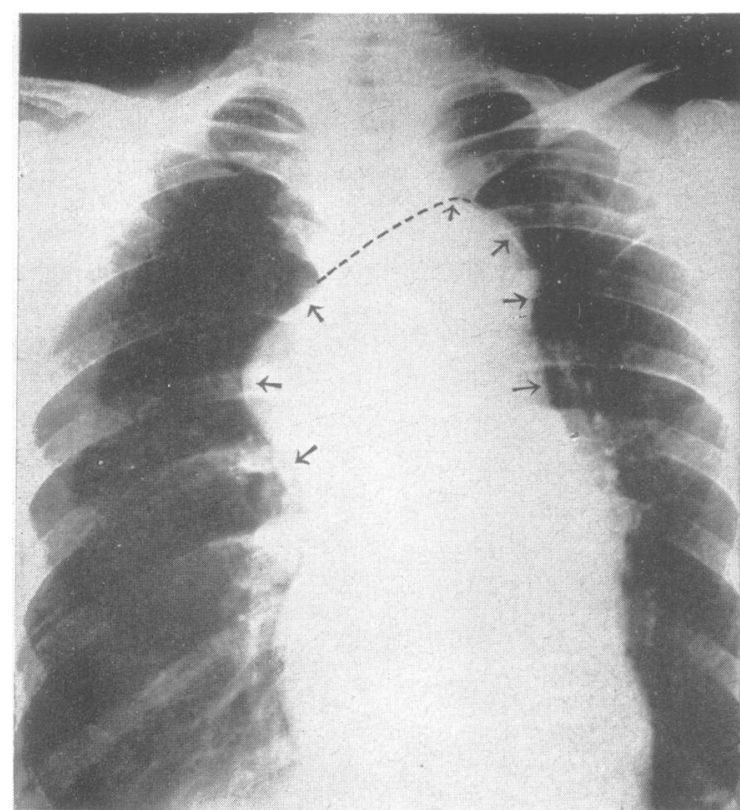

FIG. 21·(a).-Case 21, aortic arch, anterior view.

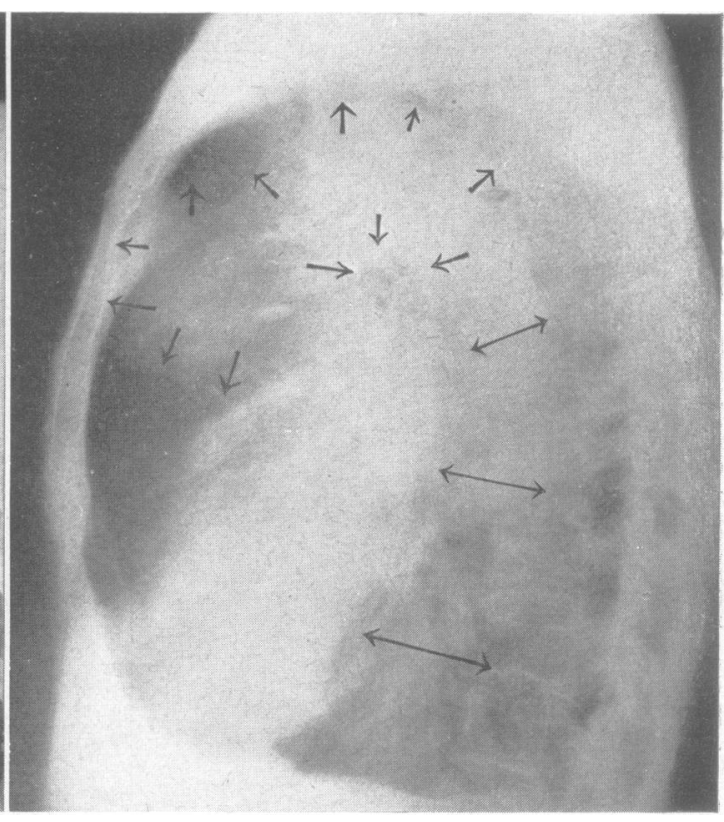

Fig. 21 (b).-Case 21, aortic arch, lateral view.

\section{Adult Soft Tissue Lesions of Syphilis}

Aortic ANEurysm.-Two cases only are shown from a series of syphilitic lesions, principally to illustrate the point that aneurysm cannot always be excluded by a routine anterior view.

Case 21. Male, aged 52.-This patient collapsed in the street and gave a vague story of dizziness and unfitness with recent loss of voice. Initially he was thought to be suffering from pneumonia, but after $x$-ray examination he was transferred to the venereal diseases department.

$X$ rays show obvious aneurysmal enlargement of the 1st and 2nd parts of the aortic arch in the anterior view (Fig. 21a) : the lateral view (Fig. 21b) demonstrates the large anterior sac from the 1st part eroding the sternum (arrows).

Calcification is visible over the arch and down the thoracic descending aorta.
Case 22. Male, aged 58.-This case is included for the sake of contrast. He had 5 months' non-specific treatment for an unrecognized luetic leg ulcer. The routine anterior chest film (Fig. 22a) taken later for the clinic shows little suspicion of aneurysm, but screening was undertaken because of an obvious left ventricular increase.

The lateral view (Fig. 22b) shows, by calcification within, how extensive the fusiform aneurysm really is (arrows).

\section{Gumma OF Lung}

Case 23. P. W., aged 49.-This patient presented with cough and chest pain. The anterior chest $x$ ray (Fig. 23a) showed a lesion overlaid by the heart apex, and tomography focused the shadow at 3 " depth from the posterior ribs (Fig. 23b).

In view of the patient's age, lung neoplasm of peripheral type was suspected in the absence of signs of tubercle. Thoracotomy revealed a granuloma which proved on section to be a gumma. 

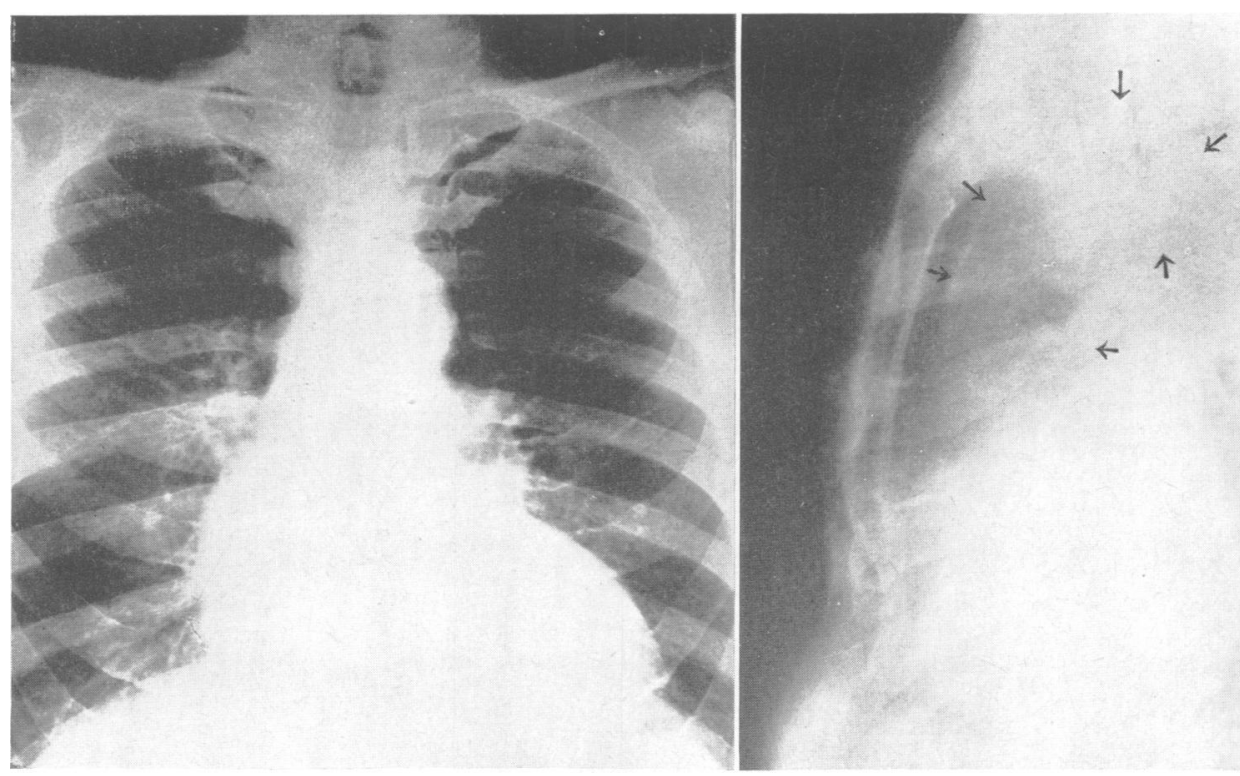

FIG. $22(b)$.-Case 22, aortic arch, lateral view.

FIG. 22 (a).-Case 22, aortic arch, anterior view.
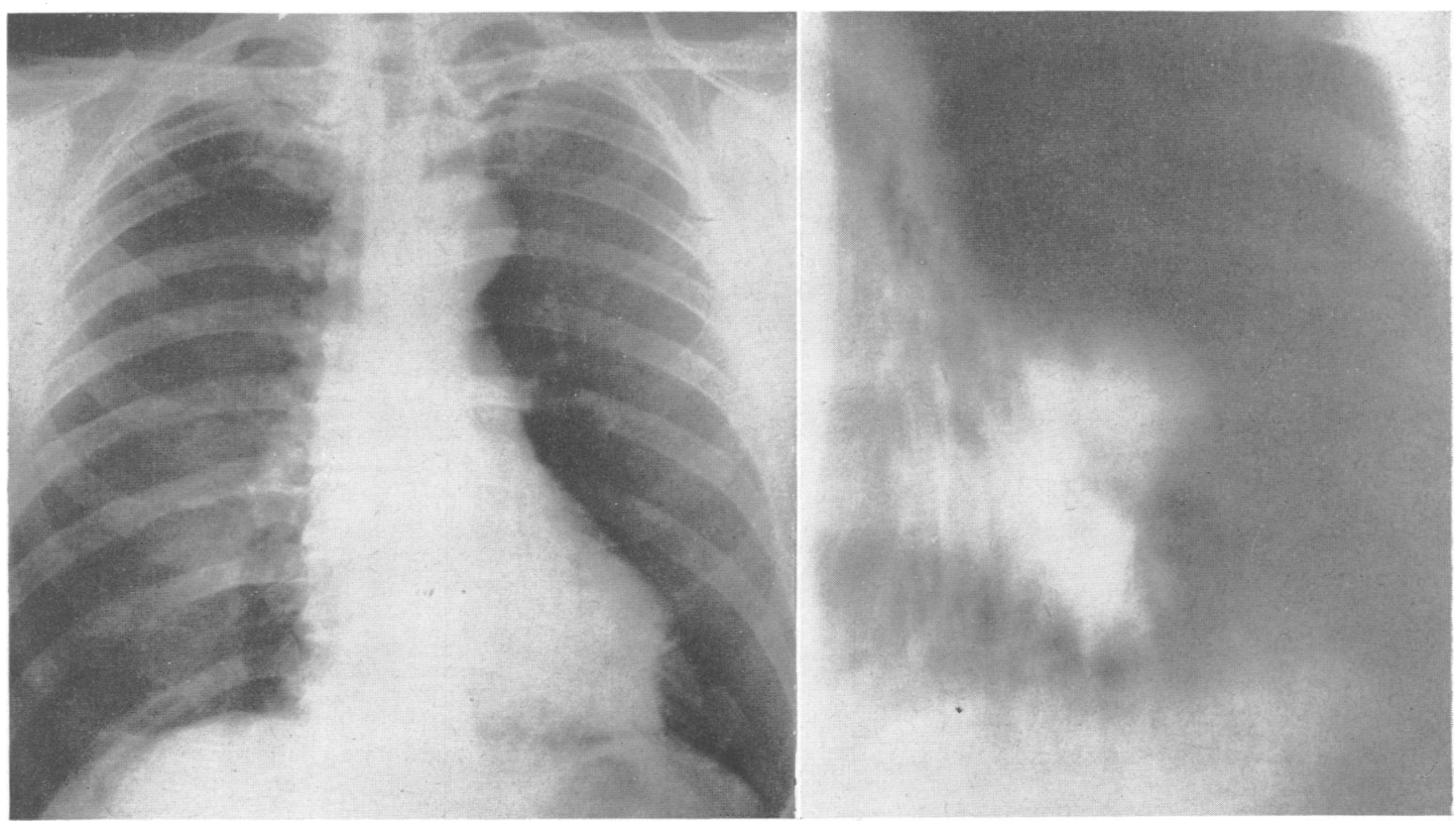

Fig. 23 (a).-Case 23, anterior chest $x$ ray.

Fig. 23 (b).-Case 23, tomograph 3" posterior cut. 


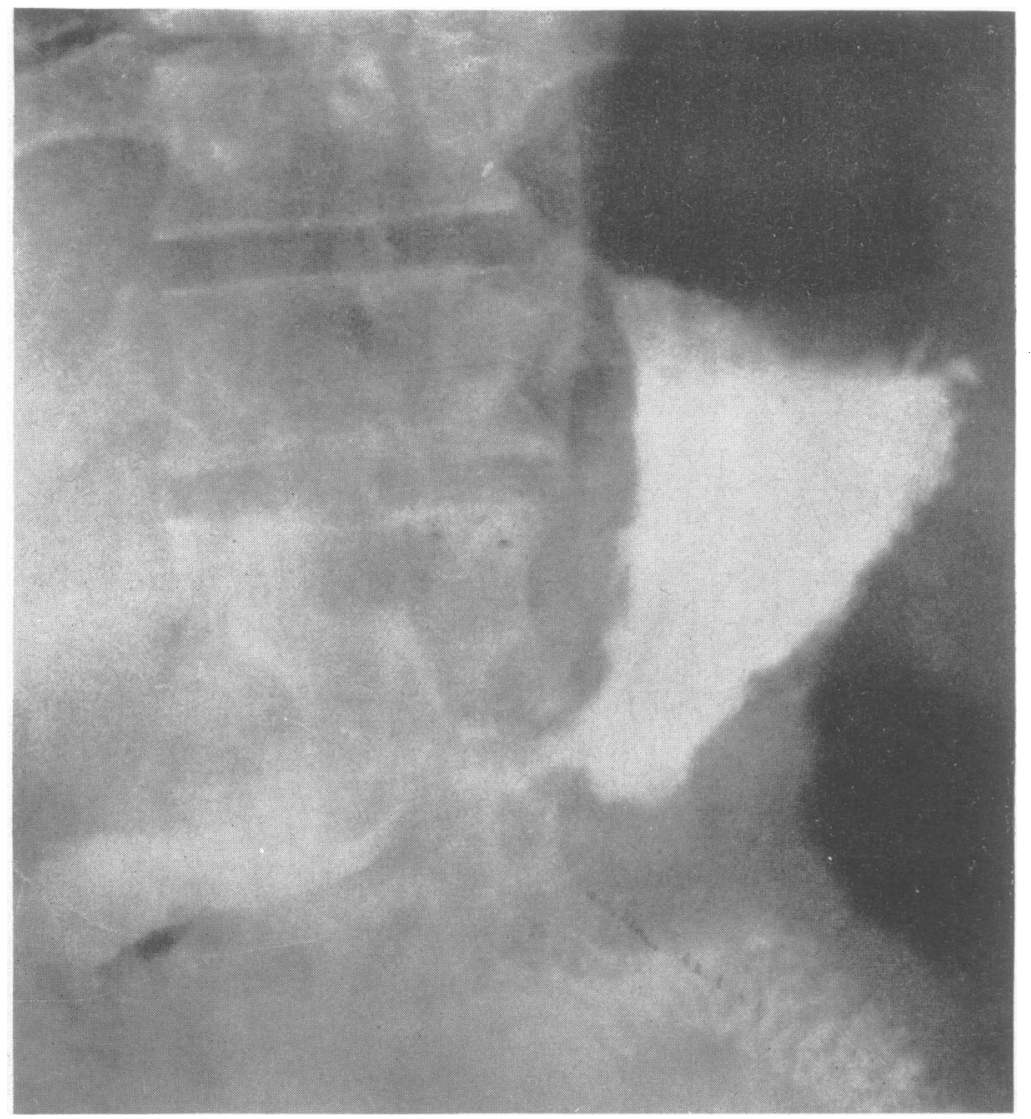

FIG. 24.-Case 24, radiograph of stomach showing filling defect.

GASTRO-INTESTINAL LeSIONS.-Gummatous involvement of the stomach may simulate the radiological features of ulcer-cancer by showing local defects of filling during a barium meal. More rarely still, a submucous infiltration may spread to indurate the organ, much as a scirrhous carcinoma more commonly does. Examples of both types of masquerade are shown below :

Case 24. T. T., aged 58 (Dr. Boulton Myles).-This patient was admitted to hospital in 1937, presenting with vomiting and ascites. Barium meal (Fig. 24) showed defective filling and narrowing of the pars media and pyloric end of the stomach. These features were reported openly in the light of experience. The Wassermann reaction proved positive. The patient was alive and well in 1947.

Case 25. I. P.-This is an example of syphilitic linitis plastica. She presented clinically as a probable case of cerebral tumour. Her complaints of vomiting of food and bile, severe occipital headaches, and dizziness, increasing over 2 weeks, were supported by the findings of choked disks with reflex disturbances. Routine Wassermann reaction proved positive. Barium meal (Fig. 25a) revealed a small rigid undistendable stomach through which barium poured continuously. No peristalsis occurred. These features characterize sub-mucous induration. After a few days of penicillin treatment vomiting stopped. A second barium examination 3 weeks after intensive treatment (Fig. 25b) now showed a normally distendable stomach which emptied gradually by peristalsis, and was pliable to palpation under the screen. Clinically, test-meal, etc., showed complete restitution to normal, and the associated neuro-syphilis also cleared up completely.

\section{LiVER AND KIDNEY Lesions}

Case 26. C. B., aged 65.-This patient was under investigation in hospital for pyuria and renal failure. Intravenous pyelography was carried out ; 15 minutes after injection of an opaque medium the radiograph (Fig. 26a) shows a non-functioning left kidney and diffuse calcareous deposits in the liver area. Further information on the left kidney was sought by retrograde 


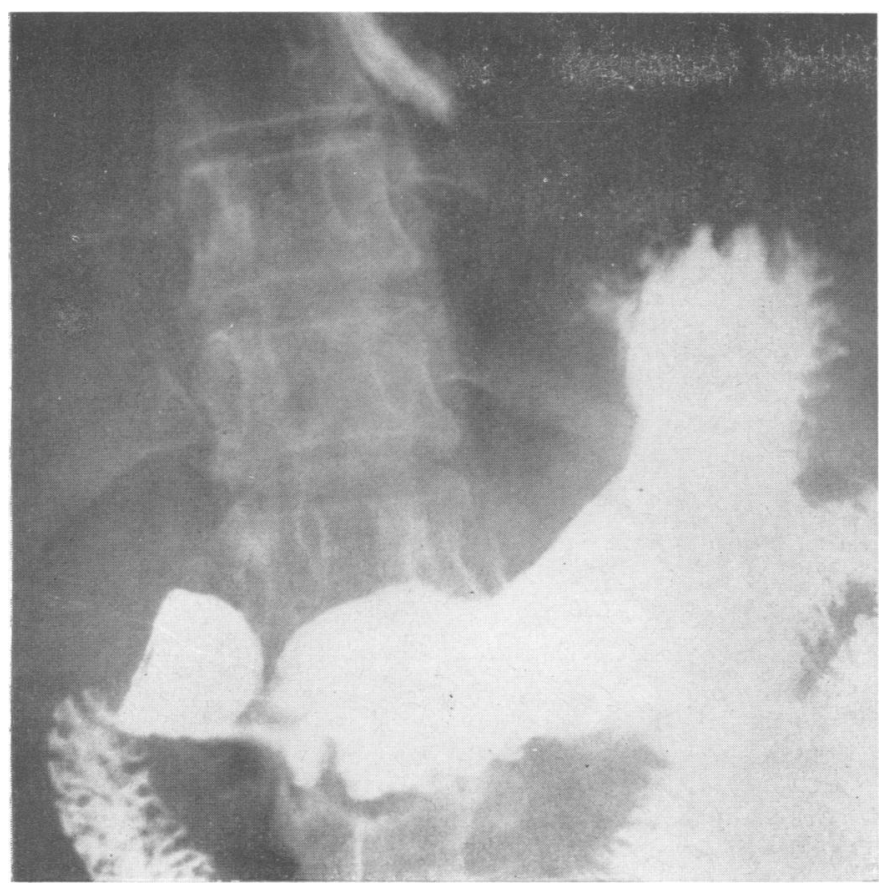

Fig. 25 (a).-Case 25 , radio graph of stomach.

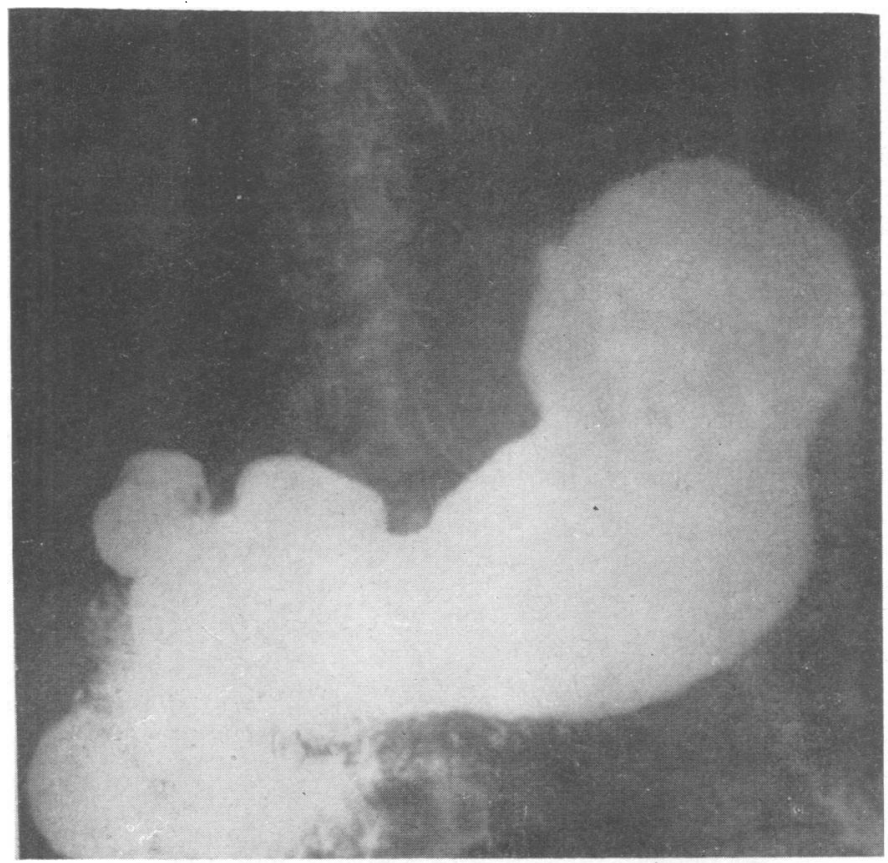

Fig. 25 (b).-Case 25, second examination after 3 weeks' intensive treatment.

pyelogram. The morphological picture (Fig. 26b) shows an ulcero-cavernous type of destruction, most commonly seen in tuberculous renal disease. In spite of the absence from the urine of the bacillus, a diagnosis of T.B. was made and this was apparently confirmed by the coexistence of liver calcifications. Post-mortem examination however revealed the gummatous nature of both liver and kidney lesions (Fig. 26a and $b$, overleaf). 
Fig. 26(b)._Case 26, left kidney ; retrograde pyelogram.

\section{Conclusion}

The majority of features encountered in cases of syphilis as seen by a practising radiologist to-day are briefly summarized above. Stress has been laid on the possibilities which have to be kept in mind in differential diagnosis, and the importance of obtaining adequately penetrating radiographs for the proper structural demonstration of dense bone. It is the pattern, and not the density, of altered bone which gives the better clue to diagnosis between bone infection, constitutional abnormalities, and neoplasms. Soft tissue calcifications, like other abnormal densities, cannot alone stigmatize a case as specific. In the lungs, the alimentary canal, the liver, and the kidneys etc., syphilis is seldom recognized during life, either clinically or radiologically. Yet it certainly occurs, and routine consideration of this possibility in differential diagnosis would undoubtedly bring more cases to light. 